\title{
Biochemical effects of a pollutant gradient - summary
}

\author{
R. F. Addison* \\ Department of Fisheries and Oceans, Bedford Institute of Oceanography, Dartmouth, Nova Scotia, Canada B2Y 4A2
}

\section{INTRODUCTION}

Biochemical measurements to evaluate the effects of pollutants on marine (or aquatic) organisms have developed considerably during the last decade. Emphasis in recent years has been to develop techniques which respond to specific types of pollutants, usually with a high degree of sensitivity. This is reflected in the techniques evaluated during the GEEP Workshop: 2 major groups of methods were applied - measurements of components of enzyme systems involved in xenobiotic metabolism, which respond to certain groups of organic pollutants, and measurements of metallothionein induction which respond specifically to certain heavy metals. Measurements were made in flounder Platichthys flesus, mussels Mytilus edulis, periwinkles Littorina littorea and in crabs Carcinus maenas.

\section{MICROSOMAL XENOBIOTIC METABOLISING SYSTEMS}

Several components of the microsomal monooxygenase enzyme system were measured in fish and molluscs. These included 'Phase 1' enzymes which facilitate the first oxidations of lipophilic substrates, exemplified by ethoxyresorufin O-de-ethylase (EROD) and benzo(a)pyrene hydroxylase (B(a)PH), and 'Phase 2 enzymes which act upon the products of oxidative metabolism. The latter are exemplified by epoxide hydrolase (EH) and glutathione S-transferase (GST). In addition, concentrations of cytochrome P-450, the complex of proteins which catalyses mono-oxygenase reactions, were measured. Finally, glutathione concentrations were determined in some samples,

- This summary was written following discussions amongst all contributors to this section since this compound is involved in the conjugation of some pollutant metabolites, and since its concentration may indicate in a more general way the response of the organism to pollutant stress.

\section{Platichthys flesus}

EROD measurements in flounder Platichthys flesus liver gave the clearest and most sensitive response to expected pollutant gradients in the field samples. EROD activities measured by 2 independent methods (Fig. 1) increased ca 7 -fold between the reference site (1) and intermediately-polluted sites (2 and 3), and ca

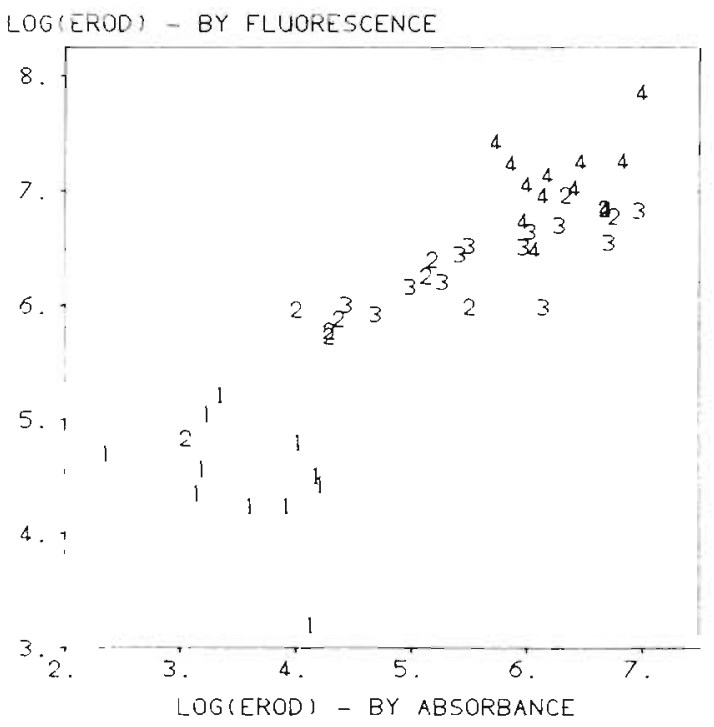

Fig. 1. Platichthys flesus. Ethoxyresorufin O-de-ethylase activity (EROD, pmol $\mathrm{min}^{-1} \mathrm{mg}$ microsomal protein ${ }^{-1}$ ) in liver of individuals from the pollution gradient (Sites 1 to 4 ) in Langesundfjord. The $x$-axis represents an absorbance measurement; The $y$-axis, fluorescence analysis; both axes are $\log _{e}$-transformed. Values are paired measurements for individuals from the sites identified $(r=0.85)$ 
15-fold between Sites 1 and 4 (the latter being expected to be the most contaminated). This trend is consistent with the observed gradient of chlorobiphenyl and, less clearly, hexachlorobenzene and octachlorostyrene concentrations in flounder liver samples, and with chlorobiphenyl and aromatic hydrocarbon burdens in Mytilus edulis (used to index the contamination gradient at these sites).

Two other measurements of mono-oxygenase activity showed similar trends, but differences between sites were less significant. $\mathrm{B}(\mathrm{a}) \mathrm{PH}$ increased about 2 - to 3 -fold between the reference site and all 3 contaminated sites, though there was no difference between $\mathrm{B}(\mathrm{a}) \mathrm{PH}$ activities in flounder from any of the contaminated sites. Cytochrome P-450 specific content showed a trend similar to that of EROD, with statistically significant differences between the sites. Other measurements - which in experimental mammalian studies have indicated mono-oxygenase induction, such as hepatic microsomal protein content or liver somatic index - revealed no consistent pattern across field sites

Concentrations of cytochrome P-450E - an isozyme associated with EROD and B(a)PH activity, and determined immunochemically - followed the same trend in field samples as did EROD activities. This provides clear evidence that the environmental chemicals to which the flounder were exposed induced a specific cytochrome P-450 isozyme whose presence was reflected in induced EROD and $\mathrm{B}(\mathrm{a}) \mathrm{PH}$ activities.

Mono-oxygenase measurements in flounder from mesocosm exposures showed no response to treatment. This could be an indication of low levels of inducing compounds, either higher molecular weight PAH or the PCBs, or it may indicate a possible effect of metal poisoning by $\mathrm{Cu}$ which was also a component of the contaminant dosing in the mesocosms. However, EROD activities in all mesocosm-exposed flounder were similar to, or slightly below, those from flounder from the field reference site. Cytochrome P-450 concentrations were also lower in mesocosm samples than in the field reference sample, and there was no evidence of induction of cytochrome P-450E. It therefore seems probable that the absence of induction reflects a low accumulation of, or exposure to, inducing compounds. This conclusion was supported by chemical analyses of tissue samples, which showed no difference in $B(a) P$ levels between treatments.

\section{Mytilus edulis, Littorina littorea and Carcinus maenas}

Consistent responses occurred in measurements of components of the mono-oxygenase system and epoxide hydrolase $(\mathrm{EH})$ in molluscs from the mesocosm experiment, although the magnitude of the changes was considerably less dramatic than those generally observed in fish. Cytochrome P-450 content of digestive gland microsomes in both mussel and periwinkle increased with increasing dose in the mesocosm exposures; a 2- to 3-fold increase was seen between control and high dose treatments. Similarly, up to a doubling of activity was seen in cytochrome c (P-450) reductase of periwinkles and $\mathrm{EH}$ of whole mussels with oil exposure in the mesocosms. In contrast, $\mathrm{B}(\mathrm{a}) \mathrm{PH}$ activity in whole mussels was elevated only in the medium dose treatment. All these observations are consistent with the results of previous studies on experimental exposures of mollusc species to hydrocarbons. In addition, the previously unstudied GST activity of the digestive gland of periwinkles was significantly (though not strongly) elevated in the high dose treatment, but no statistically significant response was seen in this enzyme in mussels; interpretation of this comparison is made difficult by the fact that a different assay was used.

In contrast to the situation in the mesocosm experiment, molluscan responses at the field sites were fewer and less consistent. Cytochrome P-450 content of the digestive gland of mussels was elevated at Site 2, as was GST activity of whole mussels. However, cytochrome P-450 content of periwinkles, and cytochrome $c$ (P-450) reductase and $\mathrm{EH}$ activities of mussels, were reduced at some of the polluted sites $(2,3$ and 4$)$. This difference between field and mesocosm data for molluscs cannot be interpreted; this indicates the very limited understanding we have at present of the basic biochemistry of these enzyme systems in such organisms. It is interesting to note the different responses of molluscs and fish to mesocosm and field exposures, which may indicate that differences in inducing agents exist for these 2 groups of organisms.

Increased crab hepatopancreas GST activity at Field sites 3 and 4 was consistent with the higher body burdens of various organic pollutants at these sites. However, the literature offers no guidance as to which of these organic contaminants (or groups of contaminants) may have caused induction. No such. differences were observed in crabs from the mesocosm exposures, suggesting that the diesel oil added to these basins was not a GST inducer.

\section{METALLOTHIONEIN}

Metallothionein (MT) measurements were made on flounder liver and kidney samples from both field and mesocosm exposures. Although the technique used would not have detected copper metallothionein ( $\mathrm{Cu}$ 
$\mathrm{MT}$ ), it did measure endogenous zinc metallothionein (Zn MT). While it is not known whether water-borne $\mathrm{Cu}$ would accumulate as $\mathrm{Cu}$ MT in the liver or kidney of the flounder, any accumulated $\mathrm{Cu}$ should have disturbed $\mathrm{Zn}$ metabolism and hence altered the levels of Zn MT

Flounder from the reference site had consistently lower MT levels than those from other sites, but in general, MT levels in individuals from the field sites were not correlated with measured metal concentrations in the same individuals. Furthermore, $\mathrm{Cu}$ was not accumulated from the mesocosm basins by experimentally-exposed fish, nor were liver or kidney MT levels elevated by the $\mathrm{Cu}$ dosing. (The lack of $\mathrm{Cu}$ accumulation in the tissues perhaps indicates that the main route of uptake is through diet rather than by direct uptake from water.)

Thus, for the present data, MT measurements on flounder liver or kidney did not reflect expected pollution gradients in the field, though it is worth noting that MT levels have reflected pollution gradients in other field situations, especially if considered in relation to measurements such as free metal concentrations in a cytosolic pool, and metals bound to high molecular weight (non-metallothionein) proteins.

Mytilus edulis samples were taken from both field sites and mesocosm exposures for MT determinations, together with total metal and high molecular weight protein-bound metal determinations. In the experimental mesocosm exposures, MT-bound Cu increased consistently with $\mathrm{Cu}$ exposure, but an even clearer effect was seen in total cytosolic protein-bound $\mathrm{Cu}$. In field samples also, total cytosolic protein-bound $\mathrm{Cu}$ appeared to be a relatively sensitive monitor of metal pollution. Finally, evidence was obtained implying that Ca homeostasis was affected in individuals from the contaminated field sites and, to a lesser extent, from the dosed mesocosm basins.

\section{CONCLUSIONS}

(1) Of the biochemical indices measured, flounder hepatic microsomal EROD activity and cytochrome P-450 isozyme concentrations showed the most sensitive response to the expected pollution gradient in the field. Since a mechanistic framework exists within which to interpret EROD and cytochrome P-450 induction in the field, in terms of the effects of body burdens of either chlorobiphenyls or PAH, these measurements can be recommended as indicators of environmental pollution by such compounds, with the reservations noted in (4) below.

(2) Measurements of cytochrome P-450 and other enzymes of xenobiotic metabolism in marine invertebrates can show responses which can be related to pollutant distribution. However, the magnitude of these responses is smaller than those seen in fish and they are more difficult to interpret because of the inadequate current knowledge of marine invertebrate xenobiotic biochemistry. Thus, these measurements are not generally applicable at present, but they should be regarded as having some potential for use in the field, when the underlying biochemistry is better understood.

(3) Several other measurements evaluated at the workshop, including cytosolic GSH measurements, tissue GST activities and flounder intestinal MFO measurements, were either insensitive or too variable to have any immediate use as biochemical indicators of the effects of pollution.

(4) It must be stressed that measurements of the sort described above cannot be applied indiscriminately. Their use requires careful evaluation, preferably in the light of complementary analyses, some of which should be chemical. The general application of such methods will require intercalibration and validation when used in different environments and for different species. 